Supplement of Biogeosciences, 18, 1241-1257, 2021 https://doi.org/10.5194/bg-18-1241-2021-supplement (C) Author(s) 2021. This work is distributed under the Creative Commons Attribution 4.0 License.

(c) (1)

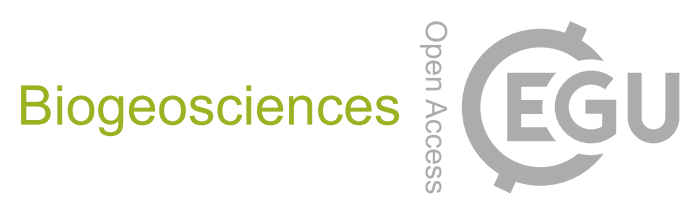

Supplement of

\title{
Age distribution, extractability, and stability of mineral-bound organic carbon in central European soils
}

Marion Schrumpf et al.

Correspondence to: Marion Schrumpf (mschrumpf@bgc-jena.mpg.de)

The copyright of individual parts of the supplement might differ from the CC BY 4.0 License. 


\section{Supplementray tables and figures.}

Table S1: Basic soil properties of the study sites (means of the three replicated samples analyzed for mineral associated carbon per soil depth with standard deviations in brackets). Values of selected cores are adopted from Schrumpf et al. 2013 (Ci: inorganic carbon, CN: OC-to-total nitrogen ratio, Feo: acid oxalate-extractable Fe, Alo: acid oxalate-extractable Al, Fed: dithionite-extractable Fe).

\begin{tabular}{|c|c|c|c|c|c|c|c|c|c|}
\hline \multirow{2}{*}{$\begin{array}{l}\text { Site and } \\
\text { soil depth }\end{array}$} & \multirow{2}{*}{$\begin{array}{l}\mathrm{pH} \\
\left(\mathrm{H}_{2} \mathrm{O}\right)\end{array}$} & OC & \multirow[t]{2}{*}{$\mathrm{CN}$} & \multirow{2}{*}{$\begin{array}{l}\mathrm{Ci} \\
\mathrm{g} \mathrm{kg}^{-1}\end{array}$} & \multirow{2}{*}{$\begin{array}{l}\text { Sand } \\
\text { g kg-1 }^{-1}\end{array}$} & \multirow{2}{*}{$\begin{array}{l}\text { Clay } \\
\text { g kg-1 }^{-1}\end{array}$} & \multirow{2}{*}{$\begin{array}{l}\text { Feo } \\
\mathrm{g} \mathrm{kg}^{-1}\end{array}$} & \multirow{2}{*}{$\begin{array}{l}\text { Fed } \\
\mathrm{g} \mathrm{kg}^{-1}\end{array}$} & \multirow{2}{*}{$\begin{array}{l}\text { Alo } \\
\text { g kg-1 }^{-1}\end{array}$} \\
\hline & & $\mathrm{g} \mathrm{kg}^{-1}$ & & & & & & & \\
\hline \multicolumn{10}{|c|}{ Hainich (Cambisol, beech) } \\
\hline $0-5$ & $6.1(0.9)$ & $73(11)$ & $13.0(0.5)$ & 0 & $22(4)$ & $546(5)$ & $3.0(1.0)$ & $14.0(0.8)$ & $1.9(1.1)$ \\
\hline $10-20$ & $6.7(0.5)$ & $27(6)$ & $10.7(0.4)$ & 0 & $28(4)$ & $514(5)$ & $2.0(0.4)$ & $15.3(1.0)$ & $1.8(0.6)$ \\
\hline $30-40$ & $7.4(0.2)$ & $11(1)$ & $9.0(0.4)$ & 0 & $23(7)$ & $731(7)$ & n.d. & n.d. & n.d. \\
\hline \multicolumn{10}{|c|}{ Hesse (Luvisol, beech) } \\
\hline $0-5$ & $4.6(0.7)$ & $31(6)$ & $13.9(0.6)$ & 0 & 68 & 342 & $1.8(0.3)$ & $11.8(1.3)$ & $1.1(0.2)$ \\
\hline $10-20$ & $4.5(0.3)$ & $14(4)$ & $11.8(0.5)$ & 0 & $56(12)$ & $315(11)$ & $1.4(0.5)$ & $11.5(1.1)$ & $1.0(0.1)$ \\
\hline $30-40$ & n.d. & $6(0)$ & $8.3(0.0)$ & 0 & $55(3)$ & $371(22)$ & n.d. & n.d. & n.d. \\
\hline \multicolumn{10}{|c|}{ Laqueuille (Andosol, grassland) } \\
\hline $0-5$ & $5.3(0.2)$ & $126(11)$ & $11.1(0.2)$ & 0 & $186(36)$ & $263(28)$ & $12.7(0.1)$ & $24.0(0.6)$ & $19.3(0.7)$ \\
\hline $10-20$ & $5.6(0.3)$ & $66(7)$ & $10.2(0.2)$ & 0 & $259(58)$ & $215(8)$ & $16.4(3.0)$ & $20.3(0.9)$ & $24.0(2.6)$ \\
\hline $30-40$ & n.d. & $50(4)$ & $10.7(0.3)$ & 0 & $236(21)$ & $225(22)$ & $15.8(1.7)$ & $20.6(1.1)$ & $27.6(2.3)$ \\
\hline \multicolumn{10}{|c|}{ Wetzstein (Podzol, spruce) } \\
\hline $0-10$ & $3.5(0.0)$ & $76(15)$ & $24.6(2.2)$ & 0 & $264(164)$ & $250(165)$ & $9.2(6.2)$ & $17.0(10.4)$ & $1.5(0.8)$ \\
\hline $10-30$ & $3.8(0.3)$ & $60(29)$ & $22.0(3.6)$ & 0 & $219(40)$ & $344(123)$ & $24.9(15.5)$ & $37.0(12.6)$ & $4.4(0.8)$ \\
\hline $30-50$ & $4.2(0.3)$ & $45(24)$ & $19.1(2.3)$ & 0 & $221(46)$ & $364(63)$ & $17.4(16.6)$ & $27.4(16.1)$ & $7.8(2.6)$ \\
\hline \multicolumn{10}{|c|}{ Gebesee (Chernozem, cropland) } \\
\hline $0-5$ & $6.8(0.1)$ & $26(2)$ & $11.3(0.9)$ & 0 & $28(5)$ & $345(5)$ & $1.4(0.1)$ & $6.9(0.3)$ & $1.3(0.1)$ \\
\hline $10-20$ & $7.0(0.4)$ & $22(2)$ & $10.5(0.1)$ & 0 & $26(3)$ & $336(2)$ & $1.4(0.2)$ & $6.9(0.3)$ & $1.4(0.1)$ \\
\hline $30-40$ & n.d. & $17(1)$ & $11.1(0.2)$ & $1.6(0.9)$ & $21(1)$ & $368(1)$ & n.d. & n.d. & n.d. \\
\hline
\end{tabular}




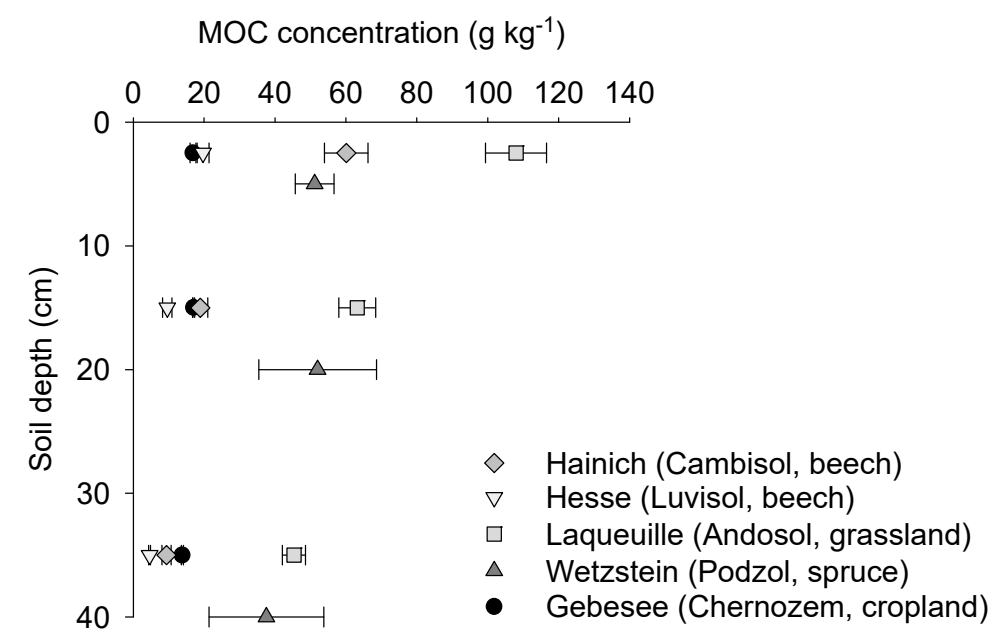

Figure S1: Original concentration of OC in mineral association (MOC, means and standard deviation) of the studied soil samples from 5 sites (data adopted from Schrumpf et al. 2013). Symbols indicate mid-points of integrated sampled depth intervals (Table 1).
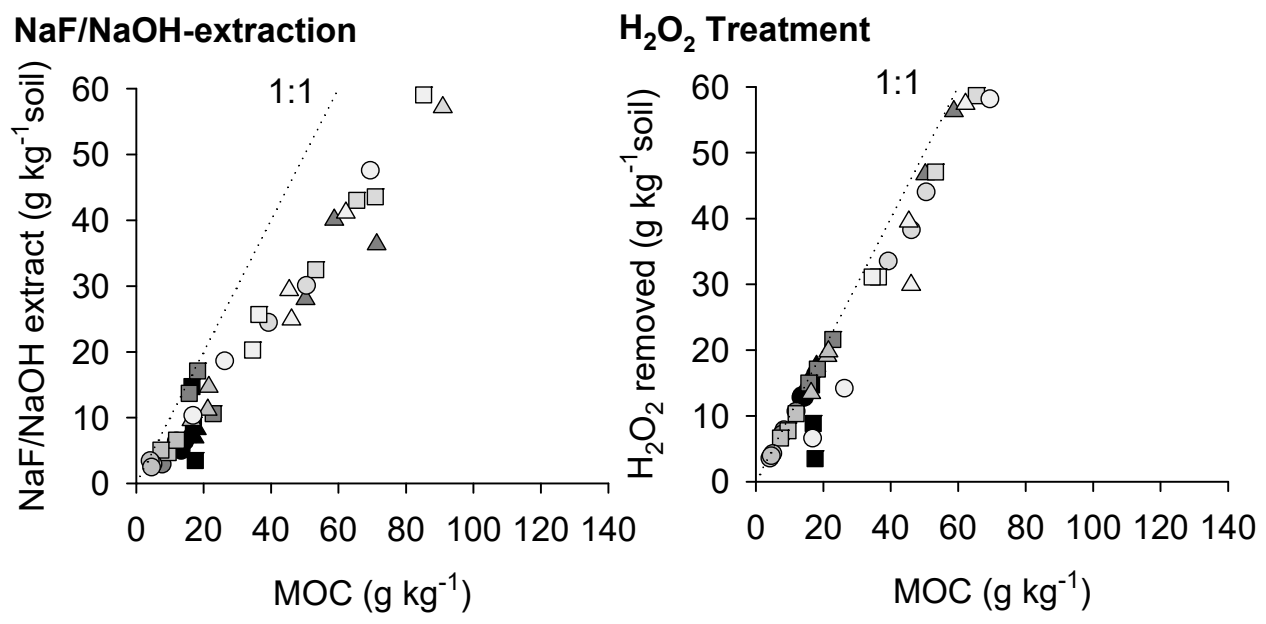

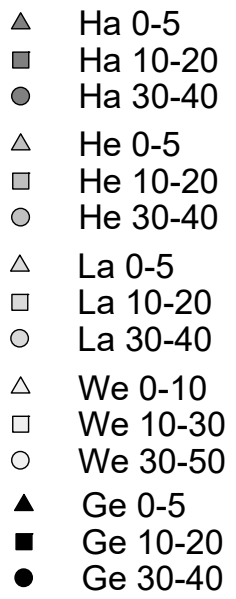

Figure S2: Dependency of removed $\mathrm{OC}$ from original concentrations of mineral-associated $\mathrm{OC}$ for the $\mathrm{NaF} / \mathrm{NaOH}$ extraction (left) or the $\mathrm{H}_{2} \mathrm{O}_{2}$ oxidation (right). 
$0-5 \mathrm{~cm}$
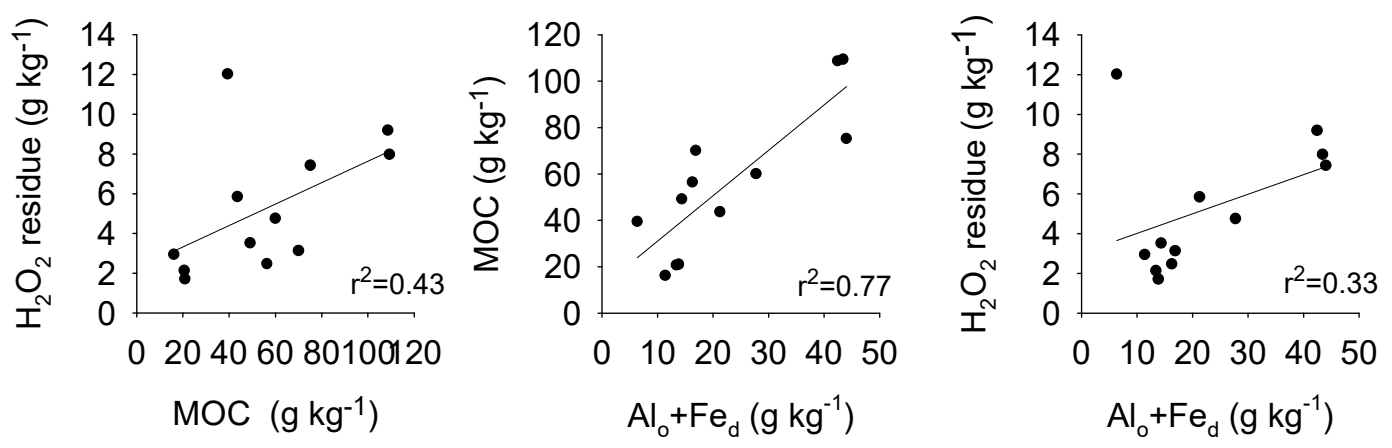

$10-20 \mathrm{~cm}$
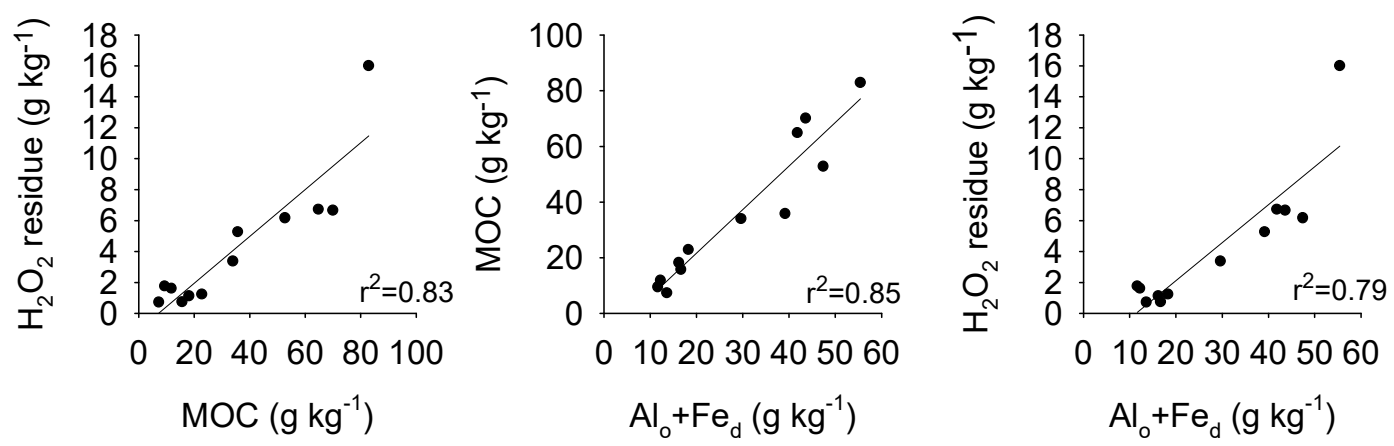

Figure S3: Relations between $\mathrm{OC}$ in mineral association (MOC), the amount of $\mathrm{OC}$ left from MOC after the $\mathrm{H}_{2} \mathrm{O}_{2}$ treatment $\left(\mathrm{H}_{2} \mathrm{O}_{2}\right.$ residue) and the content of oxalate-extractable $\mathrm{Al}\left(\mathrm{Al}_{0}\right)$ and dithionite-extractable $\mathrm{Fe}\left(\mathrm{Fe}_{\mathrm{d}}\right)$ for two soil depths $\left(\mathrm{Al}_{\mathrm{o}}\right.$ and $\mathrm{Fe}_{\mathrm{d}}$ data were taken from Schrumpf et al. (2013)).
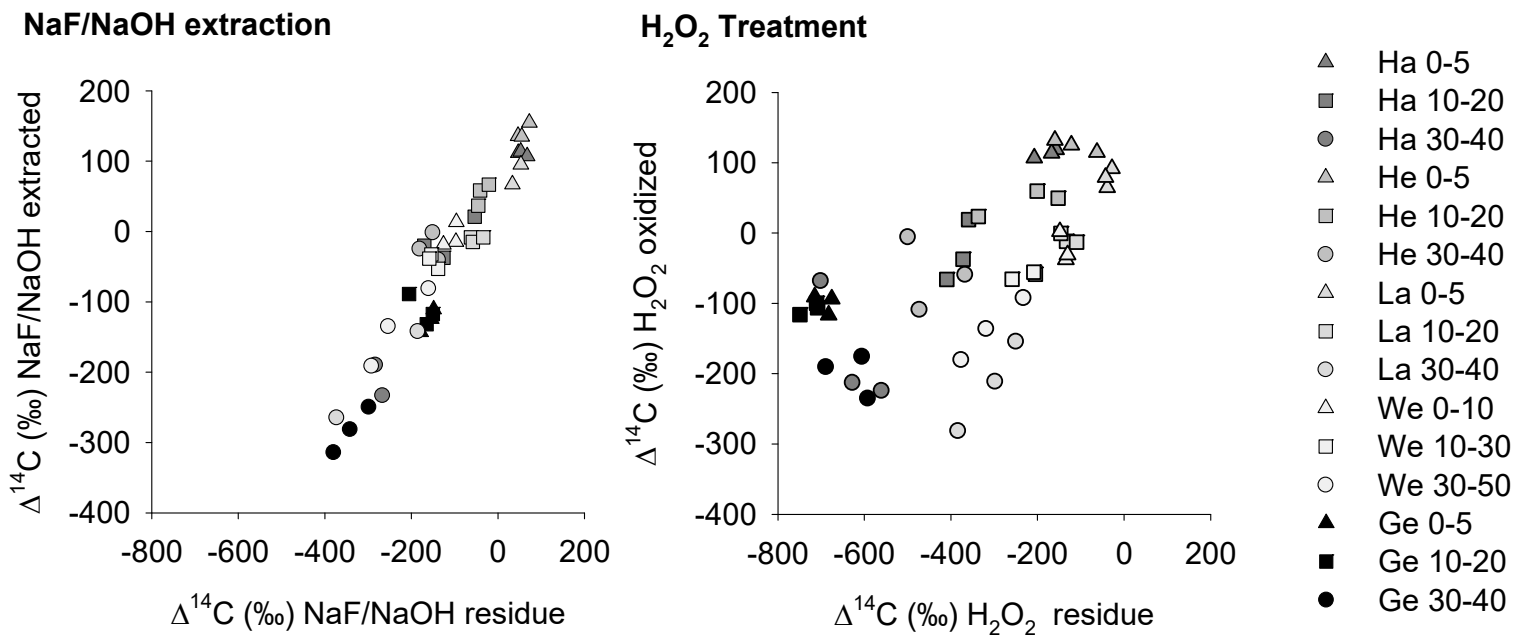

Figure S4: Correlation between ${ }^{14} \mathrm{C}$ contents of $\mathrm{NaF} / \mathrm{NaOH}$ extracts and extraction residues of $\mathrm{HF}-\mathrm{OC}$ (left) and between oxidized $\mathrm{OC}$ and residue $\mathrm{OC}$ following treatment of $\mathrm{HF}-\mathrm{OC}$ with heated $\mathrm{H}_{2} \mathrm{O}_{2}$ (right). 


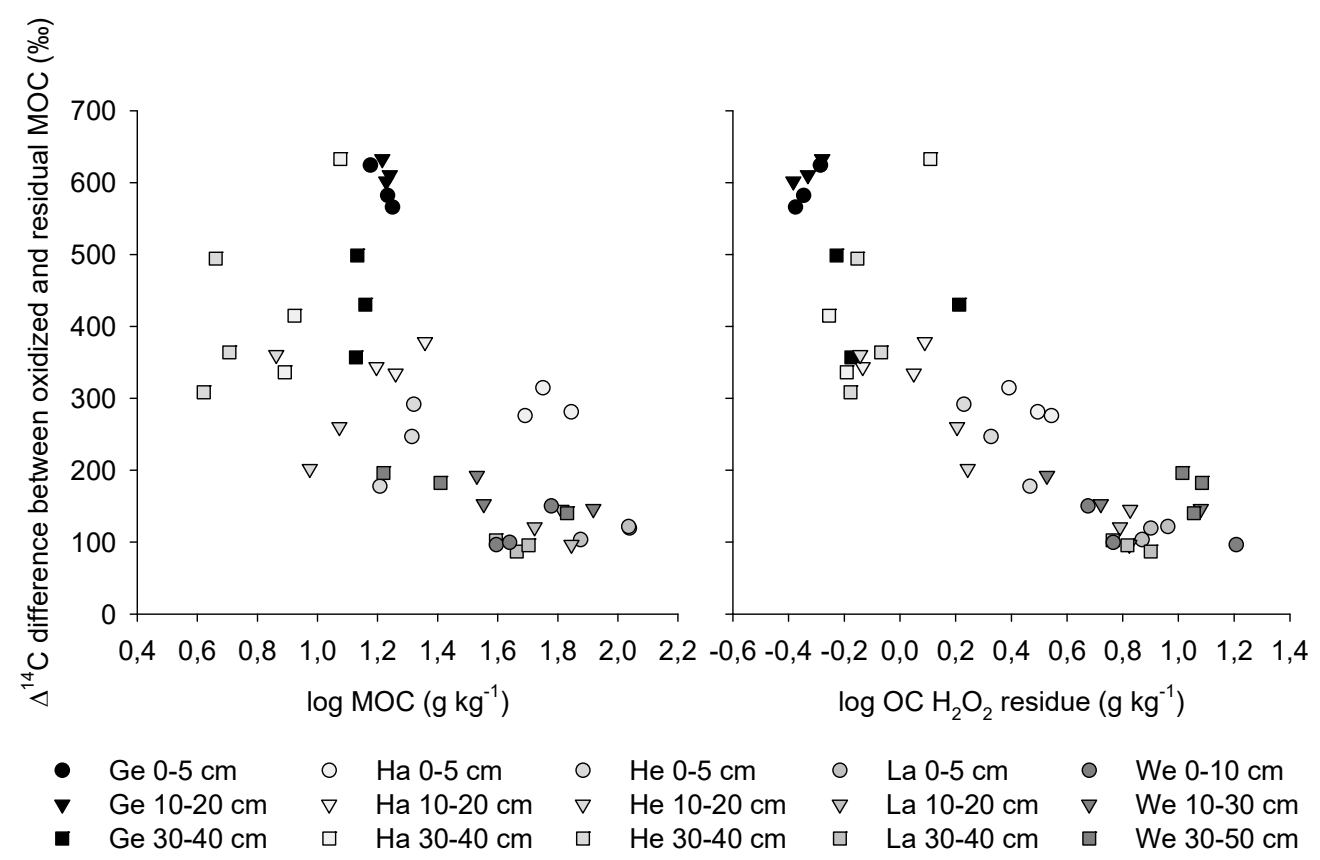

Figure S5: Decline in ${ }^{14} \mathrm{C}$ differences between oxidized and residual $\mathrm{OC}$ after $\mathrm{H}_{2} \mathrm{O}_{2}$ treatment with increasing $\mathrm{OC}$ amounts in total $\mathrm{MOC}$ and $\mathrm{OC}$ left in $\mathrm{H}_{2} \mathrm{O}_{2}$ residues across sites and depths. 

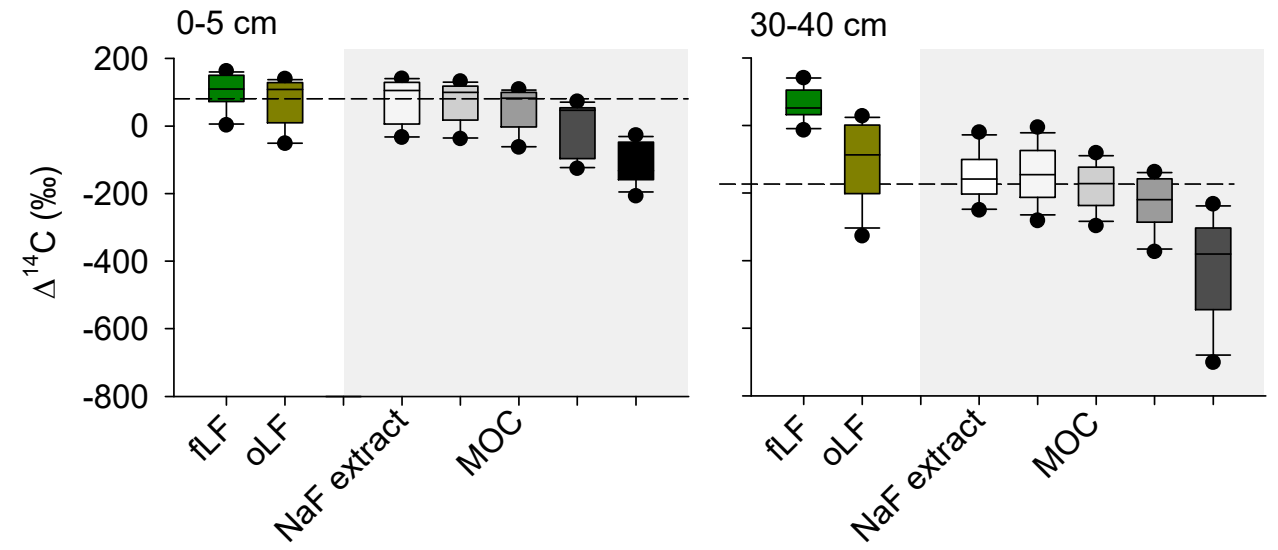

Figure S6: Box-plots giving an overview of ${ }^{14} \mathrm{C}$ contents in all $\mathrm{OC}$ fractions separated from bulk samples in 0-5 cm (left) and $30-40 \mathrm{~cm}$ (right) depth for the non-arable samples (dashed line denotes the median of the ${ }^{14} \mathrm{C}$ in bulk $\mathrm{MOC}$ ). fLF: free light fraction, oLF: occluded light fraction, fLF and oLF values adopted from Schrumpf et al. (2013). 